\title{
Using thermographic cameras to investigate eye temperature and clinical severity in depression
}

Jerome J. Maller

Shefin Sam George

Rekha Puzhavakkathumadom Viswanathan

Paul B. Fitzgerald

Paul Junor

\section{SPIE.}




\title{
Using thermographic cameras to investigate eye temperature and clinical severity in depression
}

\author{
Jerome J. Maller, ${ }^{\mathrm{a}, \star}$ Shefin Sam George, ${ }^{\mathrm{b}}$ Rekha Puzhavakkathumadom Viswanathan, ${ }^{\mathrm{b}}$ Paul B. Fitzgerald, ${ }^{\mathrm{a}}$ and \\ Paul Junor \\ ${ }^{a}$ The Alfred and Monash University Central Clinical School, Monash Alfred Psychiatry Research Centre, Melbourne, Victoria, Australia \\ baTrobe University, School of Engineering and Mathematical Sciences, Department of Electronic Engineering, Melbourne, Victoria, Australia
}

\begin{abstract}
Previous studies suggest that altered corneal temperature may be a feature of schizophrenia, but the association between major depressive disorder (MDD) and corneal temperature has yet to be assessed. The aim of this study is to investigate whether eye temperature is different among MDD patients than among healthy individuals. We used a thermographic camera to measure and compare the temperature profile across the corneas of 16 patients with MDD and 16 age- and sex-matched healthy subjects. We found that the average corneal temperature between the two groups did not differ statistically, although clinical severity correlated positively with right corneal temperature. Corneal temperature may be an indicator of clinical severity in psychiatric disorders, including depression. ( 2016 Society of Photo-Optical Instrumentation Engineers (SPIE) [DOI: 10.1117/1.JBO.21.2.026001]
\end{abstract}

Keywords: depression; thermal imaging; ocular; corneal; psychiatry.

Paper 150440R received Jul. 3, 2015; accepted for publication Dec. 30, 2015; published online Feb. 1, 2016.

\section{Introduction}

Thermoregulation is the most important and essential aspect of human homeostasis. The anterior hypothalamic nucleus and the adjacent preoptic area have been considered as the "seat of thermoregulation.", "1,2 But in recent years, it has become clear that other regions of the central nervous system (CNS), especially the spinal cord, are importantly involved in thermoregulatory processes. $^{3}$ The anterior or preoptic hypothalamus contains thermosensitive neurons that coordinate to keep the core body temperature almost constant. Preoptic neurons also receive a somatosensory input from skin and spinal thermoreceptors to indicate the peripheral temperature variations. In this way, preoptic neurons compare and integrate central and peripheral thermal information and coordinate the appropriate efferent response. Typically, input from neurons located more proximally contribute more to autonomic and metabolic responses than does input from the skin. The hypothalamus is extensively connected with different parts of the CNS, including the limbic forebrain and thus to the dopaminergic and serotonergic pathway. The hypothalamus is heavily innervated by monoaminergic and cholinergic fibers and the role of these hypothalamic neural systems in thermoregulation has been extensively investigated. ${ }^{3}$ These studies suggest that proper transmission in the dopaminergic pathway is crucial for the hypothalamus to maintain appropriate body temperature regulation to $37^{\circ} \mathrm{C}$. Due to a disturbed and hyperactive central dopaminergic transmission, patients with major depressive disorder (MDD) and schizophrenia (SCZ) are, therefore, at a higher risk of developing thermoregulatory disorders revealed by variations in core body temperature, ${ }^{4}$ as well as in superficial tissue such as cornea. Hence, classes of diseases involving abnormal dopaminergic transmission are suitable for presenting the similar temperature variation.

*Address all correspondence to: Jerome J. Maller, E-mail: jerome.maller@ monash.edu

\subsection{Methods of Measuring Core Body Temperature}

There is a definite correlation between body temperature and diseases. ${ }^{5}$ Many previous studies have analyzed core body temperature using rectal temperature although corneal, tympanic, or axillary temperatures may also indicate core body temperature. Even though rectal temperature is considered to be more accurate, the placement of a thermometer in the rectum is unpleasant and inconvenient. In humans, corneal temperature was found to be linearly related to body core temperature with corneal temperature apparently plateauing at $36.5^{\circ} \mathrm{C}$ to $37.0^{\circ} \mathrm{C}$. The corneal temperature of a healthy person is generally between $32^{\circ} \mathrm{C}$ and $35^{\circ} \mathrm{C}$, which is lower than the body temperature and higher than the atmospheric temperature. The axilla and tympanic membrane are the other easily accessible sites for temperature measurement. ${ }^{6}$ Among all the above mentioned methods, corneal temperature measurements in humans can be easily taken with relatively noninvasive procedures.

Continuous studies since 1970s have been performed to study the effect of serotonin on the thermoregulation. An overview of the evidence supporting a central role for serotoninergic neurons in the control of body temperature by the diencephalon was presented by Myers. ${ }^{7}$ Morphological analysis of the anterior hypothalamus manifested that hyperthermia was elicited in almost all species when 5-hydroxytryptophan was injected locally into this thermosensitive zone. Moreover, the heat production response during cold stress in animals such as rats and monkeys was disrupted following damage to the serotoninergic neurons in the anterior hypothalamic preoptic area. ${ }^{7}$ A study suggests that serotonin takes part in the control of the body temperature set-point. ${ }^{8}$ Some studies showed that efficacious treatment of MDD normalized the lowered amplitude of the temperature circadian rhythm and increased body temperature. For example, it was found that disturbed circadian temperature rhythm in major depression patients was restored by

$1083-3668 / 2016 / \$ 25.00$ (c) 2016 SPIE 
electroconvulsive therapy (ECT). The temperature profile over a period of $24 \mathrm{~h}$ was noticeably different in patients' pre-ECT and post-ECT and in controls. The 24-h profiles exhibited by postECT patients were similar to healthy subjects. The amplitude of circadian temperature rhythm was elevated after ECT. The mean temperatures (both asleep and 24 hours) were substantially higher in pre-ECT than post-ECT and healthy subjects. Rausch ${ }^{10}$ presented a study which claims to be the first demonstration of higher daytime body temperature in cases with major depression. The study showed that subjects with a corrected temperature above $36.8^{\circ} \mathrm{C}$ were 2.6 -fold more likely to be depressed.

\subsection{Body Temperature in Psychiatric Disorders}

Since 1930, a number of studies have been conducted to provide insight into thermoregulatory disorders in people with SCZ. A review of the relevant articles regarding thermoregulatory dysfunction in SCZ patients was conducted. ${ }^{11}$ The studies by Cameron $^{12}$ and Buck et al. ${ }^{13}$ found that SCZ patients have lower baseline temperature when compared to that of controls. The initial skin temperature recorded by Hermesh et al. ${ }^{14}$ was significantly lower in the patient group, but Douglass and Toogood $^{15}$ found that the baseline temperature in SCZ patients and controls were similar, and the study of Shiloh et al. ${ }^{16}$ showed a higher baseline temperature in patients. A longitudinal study examining the thermoregulatory alterations induced by acute antipsychotic drug (APD) administration in SCZ patients showed that acute administration of APDs decreases body temperature in drug-free $\mathrm{SCZ}$ patients by $\sim 0.36^{\circ} \mathrm{C}$, although the absolute values remain within the normal range. ${ }^{17}$

It has been reported that major depression is associated with increased core body temperature, reduced thermoregulatory cooling (e.g., sweating), and alterations in peripheral measures of serotonin (5-HT) activity, all of which are expected manifestations of impaired activity in the skin-to-brain-to-skin thermoregulatory circuit within which the ascending spinoparabrachial pathway and its CNS projections form core components. ${ }^{18}$ Furthermore, large effect size correlation was observed between reductions in Center for Epidemiologic Studies Depression Scale scores and reductions in mean core body temperatures. ${ }^{19}$

\subsection{Measurement of Corneal Temperature}

Among the array of eye imaging systems, infrared (IR) imaging is relatively noninvasive, inexpensive, and harmless, and hence widely used in medical diagnosis. Thermography (or thermal imaging) can be defined as an imaging procedure which traces the thermal sequence with an IR camera. As a part of thermoregulation, human body emits heat to the atmosphere. As the body temperature rises, the amount of energy radiated at any wavelength increases and the wavelength of peak emission decreases. An increased temperature profile indicates underlying venous flow changes. ${ }^{5}$ The temperature distribution on the surface of the body can be acquired by thermographic camera to check the health condition of a person. As the temperature variation in the anterior segment of the eye rarely occurs, it has been used to study various eye problems ${ }^{20}$ However, emissivity may also change with the water content of the eye and of the cornea. The thermographic image allows one to see variations in temperature. The visual and quantitative documentation of the temperature measurements can then be made using the appropriate software.

\subsection{Corneal Temperature in Psychiatric Disorders}

The corneal temperature of drug-free SCZ patients has been evaluated with a thermal imaging camera which demonstrated a significantly higher corneal temperature $\left(1.55^{\circ} \mathrm{C}\right.$ higher) in the patient group when compared to matched controls. ${ }^{21}$ Moreover, the study indicated that the corneal temperature has a positive correlation with the symptom severity assessed by the brief psychiatric rating scale (BPRS). Shortly afterward, a study showed that the corneal temperature of neuroleptictreated patients was significantly lower than that of the drugfree patients. ${ }^{22}$ Another study then indicated that drug-free SCZ patients have significantly higher corneal temperature when compared to that of normal healthy controls or treated patients. ${ }^{23}$ Based on these findings, Shiloh et al. conducted further studies to validate their hypothesis that the mental status of SCZ patients may be associated with their eye temperature. The stability of thermoregulatory variation in male treatment-resistant SCZ patients and healthy subjects was assessed over a period of 6 weeks; ${ }^{6}$ (to avoid changes in body temperature due to menstrual cycle, women were not included in the study). The corneal temperature was evaluated two to three times a week using a thermal imaging camera in a designated room. Shiloh et al. reported a significant correlation between SCZ patients' mean weekly PANSS scores and their mean weekly corneal temperature. $^{24}$

Drugs may alter body temperature by acting on any component of the thermoregulatory system. These components include heat production, heat conservation, and heat loss effectors and their efferent pathways, thermosensors and their afferent pathways, and neurons within the CNS that coordinate thermoregulatory effector activities. ${ }^{25}$

The current study aims at recording and investigating corneal temperature in patients with MDD and comparing them to healthy control subjects to study the relationship between corneal temperature and depression. We aimed to study the temperature profile across the cornea of the eye and to investigate the nature of corneal temperature difference in MDD patients and healthy controls. We also aimed to investigate if corneal temperature correlates to the degree of symptom severity.

\section{Materials and Methods}

\subsection{Participants}

A total of 32 subjects aged 19 to 79 years [males mean $=46.88$ $(\mathrm{SD}=17.00)$; females mean $=49.00(\mathrm{SD}=12.05)]$ comprising 16 subjects with MDD and 16 age- and sex-matched healthy controls underwent psychiatric and ocular thermographic assessments (Table 1). Subjects were recruited either through public notices or word of mouth from the clinical services of The Alfred Hospital, Victoria, Australia. MDD subjects were recruited before their transcranial magnetic stimulation (TMS) treatments began. Each was treated with either leftsided repetitive TMS (rTMS; $10 \mathrm{~Hz}$ ) or right-sided slow $(1 \mathrm{~Hz})$ TMS. MDD subjects were required to have a diagnosis of major depressive disorder as determined by a treating psychiatrist and confirmed with the Mini-International Neuropsychiatric Interview $(\mathrm{MINI})^{26}$ and a score on the Hamilton Depression Rating Scale (HAMD) ${ }^{27}$ of at least 13, representing moderate-to-severe depression. Exclusion criteria included another current or previous DSM-IV axis I diagnosis, current active medical problem, and known neurological disease. In addition, control subjects were required to have no 
Table 1 Demographics of the patient and control subjects.

\begin{tabular}{lcc} 
& \multicolumn{2}{c}{ Group } \\
\cline { 2 - 3 } Variable & MDD & Control \\
\hline Number (M:F) & $16(8: 8)$ & $16(8: 8)$ \\
Years of age mean & $48.31 \pm 12.72$ & $47.56 \pm 16.57$ \\
HAMD mean & $25.06 \pm 5.08$ & $\mathrm{~N} / \mathrm{A}$ \\
& Range: 13 to 32 & \\
\hline
\end{tabular}

Note: \pm , SD; M, males; F, females; MDD, major depressive disorder; $\mathrm{N} / \mathrm{A}$, not applicable; HAMD, Hamilton depression rating scale.

history of psychiatric illness as screened by the psychiatric assessment schedule. ${ }^{28}$ All subjects provided written informed consent on a form approved by the Alfred Hospital, La Trobe University, and Monash University Human Subjects Research and Ethics Committees.

Patients were taking a variety of medications, including SSRIs, SNRIs, MAOIs, and atypical antipsychotics.

No subjects had diagnosed eye disease (e.g., glaucoma and cataracts).

\subsection{Thermographic Camera}

Because the normal core temperature of a human body ranges between $32^{\circ} \mathrm{C}$ and $38^{\circ} \mathrm{C}$, the temperature measurement range required for this study is small. We used an NEC F30S Thermo Shot (NEC Corporation, Japan) which has a spatial resolution of $160 \times 120$ pixels, thermal resolution of $100 \mathrm{mK}$ $\left( \pm 0.1^{\circ} \mathrm{C}\right)$, and accuracy of $\sim 2000 \mathrm{mK}\left( \pm 2^{\circ} \mathrm{C}\right)$. Emissivity was set to 0.98 based on previous corneal temperature studies. $^{29-31}$

\subsection{Room Temperature, Humidity and Lux Monitoring, and Corneal Thermographic Acquisition}

All thermographic assessments were performed in a specified designated room with a temperature of $23.2^{\circ} \mathrm{C}$ to $26.4^{\circ} \mathrm{C}$ and relative humidity of $42 \%$ to $47 \%$. Based on the specifications of our camera, the best measurement distance to image each cornea separately was evaluated to be $20 \mathrm{~cm}$. We constructed apparatus to measure and record the temperature, humidity, and intensity of light at all times.

A National Instruments USB-6009 data acquisition device was used to acquire differential mode data from temperature (LM35; Texas Instruments), humidity (HIH 4000; Honeywell S\&C), and light sensors (ORP12; Silonex, UK). LabVIEW (National Instruments, Texas), and an open standard graphical programming environment was used to access and control the data acquisition hardware.

Both eyes were imaged separately. Using a protractor, the images were taken at an angle of $15 \mathrm{deg}$ between the eye and the camera to avoid variations in surface emissivity at the given observation angle. Moreover, the human eye has an ellipsoidal-type shape, so some variations and errors can occur in the IR image due to variation in the observation angle. By fixing the viewing angle, variations due to surface emissivity and observation angle were avoided. GORATEC Thermography Studio (GORATEC Technology, Erding, Germany) was used to provide an accurate detailed analysis of the images stored into the memory card. The analysis objects such as points, lines, and areas helped to create histograms and line profiles to present the thermal information contained by the images. To study the thermal information of the corneal surface, an elliptical area was used because it resembles the oval shape of eye. This area was selected manually by comparing with the RGB image provided by the camera and visual inspection of the temperature profile of the eye. The area of the ellipse (the physical imaging area) was fixed at 710 to $715 \mathrm{px}^{2}\left(35,400.42 \mathrm{~mm}^{2}\right)$ to ensure uniform analysis in all participants; regional areas were one quarter of the total area (i.e., quadrants). An area histogram showed the temperature distribution, indicating the minimum, maximum, average, and radiation power values across the cornea. The temperature profiles across the cornea were created using line objects in which the line profile (the length of which depends upon the area of the full eye) showed temperatures of every point along it. This indicated whether the temperature profile across the cornea of a depression patient was different from healthy controls.

\subsection{Procedure}

Each participant participated in a single $1.5 \mathrm{~h}$ session which consisted of a cognitive and symptom rating session (including administration of the DSM-IV, MINI, and HDRS to patients with major depression disorder) of $\sim 45 \mathrm{~min}$, and a thermographic assessment of $\sim 25$ to $30 \mathrm{~min}$. For the thermographic component of the study, participants were required to sit in a comfortable chair for $\sim 15$ min with their chin stabilized in a chin-holder and eyes wide open. Participants were asked not to blink during the image acquisition periods. The thermographic camera (mounted on a tripod) then imaged various sections of each cornea to estimate their temperatures. The images were stored in JPEG format containing the radiometric data for analysis. The ambient temperature, humidity, and light intensity of the room were recorded so that they could be considered when calculating the ocular temperatures.

\subsection{Statistical Analysis}

GORATEC Thermography Studio and LABView were used for detailed analysis and evaluation of thermal images. SPSS for Windows version 22.0 (SPSS, Illinois) was used to conduct statistical analyses between the groups. Analyses were two-tailed and were evaluated for significance at the 0.05 alpha level. Simple chi-square and analysis of variance (ANOVA) were employed to compare demographics and ocular temperatures, and Pearson correlations were used to investigate the association between clinical severity and ocular temperature.

\section{Results}

\subsection{Demographics}

There was no significant age or gender difference among the groups, hence combined male and female results are presented. Mean HAMD was 25.06 ( $\mathrm{SD}=5.08$ ); two patients had scores below 20 (moderate depression), while the remaining 14 patients had scores beyond 20 (major depression). 


\subsection{Corneal Temperature in Major Depressive Disorder and Controls}

There were no significant differences in mean corneal temperatures between the MDD patients and control subjects [right cornea: $F(1,30)=0.624, p=0.436$; left cornea: $(F(1,30)=$ $0.664, p=0.664$; Table 2]. There was no significant difference in temperature between the left eye and right eye of patients with MDD or in control subjects. Controlling for age made no difference to these results. Figure 1 shows an example of temperature measurement.

\subsection{Corneal Temperature and Clinical Severity}

There was no significant correlation between HAMD and age $[r(16)=0.87, p=0.748]$. All correlations between age and

Table 2 Mean ocular temperatures of the patient and control groups.

\begin{tabular}{|c|c|c|}
\hline \multirow[b]{2}{*}{ Variable } & \multicolumn{2}{|c|}{ Group } \\
\hline & MDD & Control \\
\hline \multicolumn{3}{|c|}{ Right } \\
\hline \multirow[t]{2}{*}{ Cornea } & $33.86 \pm 2.58$ & $34.606 \pm 2.33$ \\
\hline & Range: 30.80 to 39.40 & Range: 31.30 to 39.50 \\
\hline Quadrant A & $33.83 \pm 2.60$ & $34.53 \pm 2.31$ \\
\hline Quadrant B & $33.66 \pm 2.60$ & $34.39 \pm 2.37$ \\
\hline Quadrant C & $33.73 \pm 2.57$ & $34.44 \pm 2.35$ \\
\hline Quadrant D & $33.74 \pm 2.57$ & $34.51 \pm 2.34$ \\
\hline Entire eyeball & $33.58 \pm 2.56$ & $34.26 \pm 2.31$ \\
\hline \multicolumn{3}{|c|}{ Left } \\
\hline Cornea & $33.23 \pm 1.74$ & $33.03 \pm 1.19$ \\
\hline Quadrant A & $33.18 \pm 1.76$ & $32.99 \pm 1.22$ \\
\hline Quadrant B & $33.06 \pm 1.78$ & $32.84 \pm 1.19$ \\
\hline Quadrant C & $33.09 \pm 1.78$ & $32.93 \pm 1.21$ \\
\hline Quadrant D & $33.11 \pm 1.76$ & $32.89 \pm 1.48$ \\
\hline Entire eyeball & $32.92 \pm 1.78$ & $32.68 \pm 1.23$ \\
\hline
\end{tabular}

Note: \pm , SD. all ocular temperatures measurements were negative and none were statistically significant (all $p>0.05$ ). There were statistically significant positive correlations between right eye temperatures and clinical depression severity (Table 3 and Fig. 2). Controlling for age made very little difference to these results.

\subsection{Corneal Temperature and Medication}

Each patient was taking different medications, so statistical analysis of the potential effect of medication could not be clearly considered. However, two patients who were taking lithium carbonate had corneal temperatures that were among the lowest within the clinical group.

\section{Discussion}

This study reports a unique finding of right-sided ocular temperature being correlated positively with increased depression severity.

Our patients with MDD were recruited before their TMS treatments began. The protocols used have shown that those with MDD usually have an overactive (hyperactive) rightsided prefrontal cortex and underactive (hypoactive) left-sided prefrontal cortex. ${ }^{32}$ Hence, right-sided perfusion would be expected to be increased and left-sided perfusion to be decreased before treatment. Therefore, right-sided perfusion of the vessel feeding the eye (i.e., ophthalmic artery) would also be increased, resulting in increased ocular temperature. Our finding of rightsided ocular temperature being positively correlated with increased depression severity supports this assumption. This finding is consistent with previously reported findings ${ }^{21}$ which indicated that the corneal temperature has a positive correlation with the symptomatic SCZ severity assessed by the BPRS and PANSS. ${ }^{24}$

The mean ocular temperatures in this study are well within the range previously reported in large samples. ${ }^{20,33}$ Our findings are consistent with a previous study which reported a large effect size correlation between reductions in depression scores and reductions in mean core body temperatures. ${ }^{19}$ In line with this, it has been suggested that cold showers be adapted as a potential treatment for depression. ${ }^{34}$

It has also been reported that nocturnal temperature is significantly higher in depressed patients compared to controls, and decreases appreciably on recovery. ${ }^{35}$ In that study, the nocturnal sweat rates of depressed patients did not differ significantly from those of controls, but decreased significantly with recovery.

Vasodilatation and vasoconstriction after local application of vasoactive substances have shown an increase or a decrease in corneal temperature, respectively. ${ }^{30}$ Peripheral blood flow may show parallel changes with blood flow variation in the eye. ${ }^{36,37}$
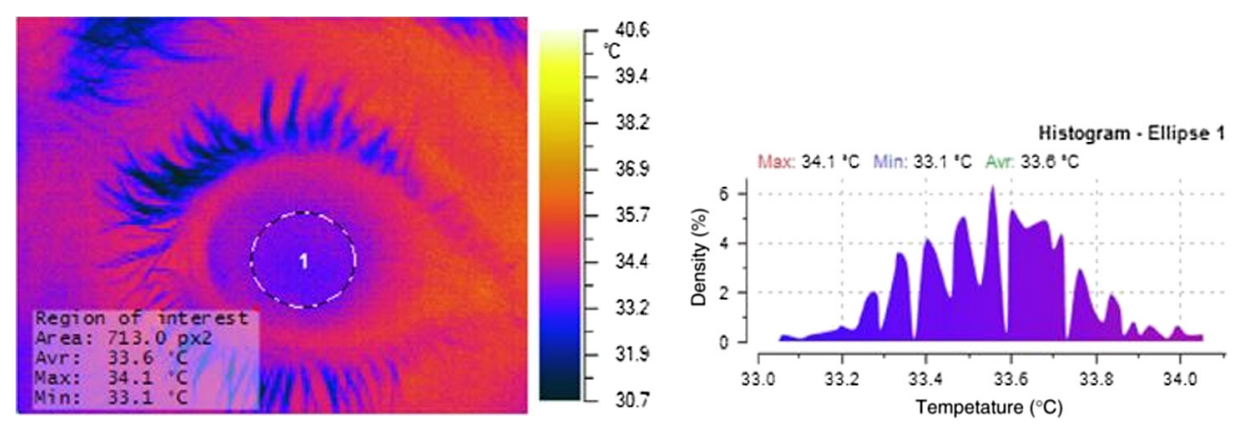

Fig. 1 Example of thermographic software used to estimate corneal temperature. 
Table 3 Correlations between HAMD and ocular temperatures.

\begin{tabular}{|c|c|c|c|c|c|c|c|c|c|c|c|}
\hline \multicolumn{6}{|c|}{ Right } & \multicolumn{6}{|c|}{ Left } \\
\hline Full & Q-A & Q-B & Q-C & Q-D & ROI & Full & Q-A & Q-B & $Q-C$ & Q-D & $\mathrm{ROI}$ \\
\hline $0.537^{\star}$ & $0.538^{*}$ & $0.537^{\star}$ & $0.535^{\star}$ & $0.539^{*}$ & $0.539^{*}$ & 0.260 & 0.256 & 0.237 & 0.244 & 0.240 & 0.212 \\
\hline
\end{tabular}

Note: *denotes $p<0.05 . Q$, Quadrant.

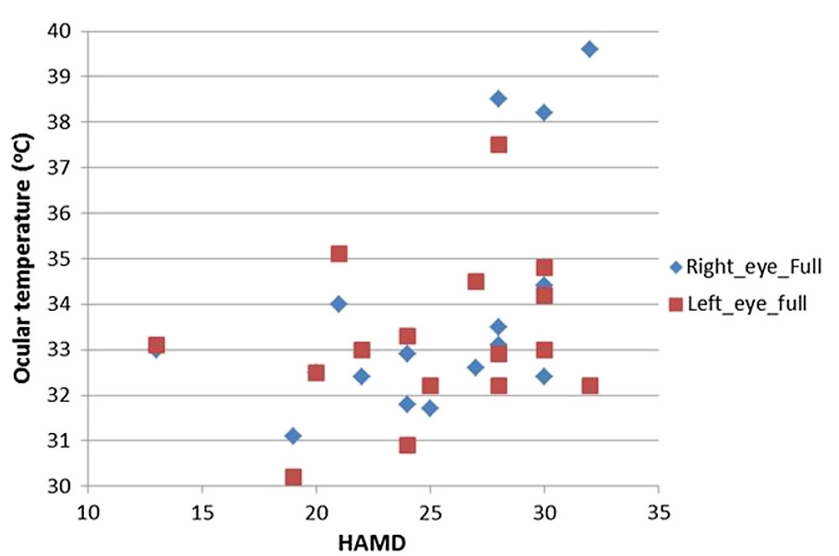

Fig. 2 Relationship between HAMD scores and ocular temperatures (whole eye averages).

This preliminary study indicated that in a controlled environment, the average corneal temperature of patients with MDD was no different than that of healthy control subjects. However, we observed that the corneal temperature of patients undergoing lithium medication were among the lowest of all the subjects. While it is difficult to conclude that reduced corneal temperatures were related to the effect of lithium medication, it does lend support to the premise that lithium treatment is hypothermic. ${ }^{38}$ Future investigations with larger groups of subjects may be able to address the extent to which medication impacts upon corneal temperature.

Future investigations should consider multimodality imaging. For example, merging MRI/CT with thermography may enable better quality and fidelity, especially for medical applications in which the temperature changes are clinically significant. $^{39}$

\subsection{Limitations}

The study was limited by the number of participants, hence the results must be interpreted with caution. The study was also limited by the accuracy of the thermographic camera which may be insufficient for this study, noting that the temperature difference between MDD and normal groups may be beyond the thermal resolution of our camera. Therefore, the average corneal temperature of the patients with MDD may be found to be different from that of the healthy controls in more extensive studies recruiting higher numbers of subjects and using cameras of higher thermal resolution. Corneal temperature evaluation may then potentially serve as a noninvasive diagnostic tool to discriminate MDD patients from normal healthy people.

Measurements were not acquired at the same time of day for each subject, and ocular temperatures fluctuate during the day. ${ }^{40}$ We conducted only one measurement per person, so we did not account for this diurnal variability. Furthermore, body temperature (and, therefore, ocular temperature) also varies with menstrual cycle. ${ }^{41}$ These factors may have impacted upon the reliability of our conclusions. Hence, we will endeavor to control for these variables in any future corneal temperature investigations.

As this was a cross-sectional study, we could not establish a direct cause-consequence relationship between corneal temperature and depression severity.

\section{Conclusion}

This study, conducted in a room with a controlled environment, showed no difference between mean corneal temperatures of MDD patients and matched controls. The main finding is that corneal temperature is significantly related to depression severity, and future research in larger samples will facilitate our understanding of this association. The possible effects of medication on thermoregulatory functions can be studied by analyzing drug-free and -treated depression patients separately. Awareness of the medication history of the treated patients can inform a better understanding of the effects of antidepressants on their body temperature.

\section{Acknowledgments}

The authors thank all the patients and volunteers who agreed to participate in this study.

\section{References}

1. B. Monge-Roffarello et al., "The medial preoptic nucleus as a site of the thermogenic and metabolic actions of melanotan II in male rats," Am. J. Physiol. 307(2), R158-R166 (2014).

2. D. Martelli et al., "The direct cooling of the preoptic-hypothalamic area elicits the release of thyroid stimulating hormone during wakefulness but not during REM sleep," PLoS One 9(2), e87793 (2014).

3. R. M. Lopachin and T. A. Rudy, "The thermoregulatory effects of noradrenaline, serotonin and carbachol injected into the rat spinal subarachnoid space," J. Physiol. 333, 511-529 (1982).

4. S. Elsenga and R. H. Van den Hoofdakker, "Body core temperature and depression during total sleep deprivation in depressives," Biol. Psychiatry 24(5), 531-540 (1988).

5. S. Bagavathiappan et al., "Infrared thermal imaging for detection of peripheral vascular disorders," J. Med. Phys. 34(1), 43-47 (2009).

6. C. G. Blainey, "Site selection in taking body temperature," Am. J. Nursing 74(10), 1859-1861 (1974).

7. R. D. Myers, "Serotonin and thermoregulation: old and new views," $J$. Physiol. 77(2-3), 505-513 (1981).

8. V. D. Sitnikov et al., "[Effect of serotonin on thermoregulation in normothermic hibernators and during arousal from deep hypothermia]," Biull. Eksp. Biol. Med. 101(1), 5-7 (1986).

9. M. P. Szuba, B. H. Guze, and L. R. Baxter Jr., "Electroconvulsive therapy increases circadian amplitude and lowers core body temperature in depressed subjects," Biol. Psychiatry 42(12), 1130-1137 (1997).

10. J. L. Rausch et al., "Depressed patients have higher body temperature: 5-HT transporter long promoter region effects," Neuropsychobiology 47(3), 120-127 (2003). 
11. T. W. Chong and D. J. Castle, "Layer upon layer: thermoregulation in schizophrenia," Schizophrenia Res. 69(2-3), 149-157 (2004).

12. D. E. Cameron, "Heat production and heat control in the schizophrenic reaction," Arch. Neurol. Psychiatry 32, 704-711 (1934).

13. C. W. Buck, H. B. Carscallen, and G. E. Hobbs, "Temperature regulation in schizophrenia. I. Comparison of schizophrenic and normal subjects. II. Analysis by duration of psychosis," AMA Arch. Neurol. Psychiatry 64(6), 828-842 (1950).

14. H. Hermesh et al., "Heat intolerance in patients with chronic schizophrenia maintained with antipsychotic drugs," Am. J. Psychiatry 157(8), 1327-1329 (2000).

15. A. B. Douglass and R. W. Toogood, "Temperature regulation and dopamine in schizophrenia," Biol. Psychiatry 22(8), 1048-1050 (1987).

16. R. Shiloh et al., "Abnormal thermoregulation in drug-free male schizophrenia patients," Eur. Neuropsychopharmacolog. 11, 285-288 (2001).

17. R. Shiloh et al., "Acute antipsychotic drug administration lowers body temperature in drug-free male schizophrenic patients," Eur. Neuropsychopharmacolog. 10(6), 443-445 (2000).

18. M. W. Hale, C. L. Raison, and C. A. Lowry, "Integrative physiology of depression and antidepressant drug action: implications for serotonergic mechanisms of action and novel therapeutic strategies for treatment of depression," Pharmacol. Ther. 137(1), 108-118 (2013).

19. K. U. Hanusch et al., "Whole-body hyperthermia for the treatment of major depression: associations with thermoregulatory cooling," Am. J. Psychiatry 170(7), 802-804 (2013).

20. R. Acharya et al., "Analysis of normal human eye with different age groups using infrared images," J. Med. Syst. 33, 207-213 (2009).

21. R. Shiloh et al., "Increased corneal temperature in drug-free male schizophrenia patients," Eur. Neuropsychopharmacolog. 13(1), 49-52 (2003).

22. R. Shiloh et al., "Lower corneal temperature in neuroleptic-treated vs. drug-free schizophrenia patients," Neuropsychobiology 48(1), 1-4 (2003).

23. R. Shiloh et al., "Corneal temperature in schizophrenia patients," Int. J. Neuropsychopharmacolog. 8(4), 537-547 (2005).

24. R. Shiloh et al., "Association between corneal temperature and mental status of treatment-resistant schizophrenia inpatients," Eur. Neuropsychopharmacolog. 19(9), 654-658 (2009).

25. W. G. Clark, "Changes in body temperature after administration of amino acids, peptides, dopamine, neuroleptics and related agents," Neurosci. Biobehav. Rev. 3(4), 179-231 (1979).

26. D. V. Sheehan et al., "The Mini-International Neuropsychiatric Interview (M.I.N.I.): the development and validation of a structured diagnostic psychiatric interview for DSM-IV and ICD-10," J. Clin. Psychiatry 59(Suppl. 20), 22-33 (1998).

27. M. Hamilton, "A rating scale for depression," J. Neurol. Neurosurg. Psychiatry 23, 56-62 (1960).

28. L. Gask, The Psychiatric Assessment Schedule (revised), Department of Psychiatry, Manchester University, Manchester (1988).

29. R. Mapstone, "Determinants of corneal temperature," Br. J. Ophthalmol. 52(10), 729-741 (1968).

30. F. Girardin et al., "Relationship between corneal temperature and finger temperature," Arch. Ophthalmol. 117(2), 166-169 (1999).

31. P. Y. Hwang, P. M. Lewis, and J. J. Maller, "Use of intracranial and ocular thermography before and after arteriovenous malformation excision," J. Biomed. Opt. 19(11), 110503 (2014).

32. P. B. Fitzgerald et al., "A meta-analytic study of changes in brain activation in depression," Hum. Brain Mapp. 29(6), 683-695 (2008).
33. L. Tan, Z. Q. Cai, and N. S. Lai, "Accuracy and sensitivity of the dynamic ocular thermography and inter-subjects ocular surface temperature (OST) in Chinese young adults," Contact Lens Anterior Eye 32(2), 78-83 (2009).

34. N. A. Shevchuk, "Adapted cold shower as a potential treatment for depression," Med. Hypotheses 70(5), 995-1001 (2008).

35. D. H. Avery et al., "Nocturnal sweating and temperature in depression," Acta Psychiatr. Scand. 100(4), 295-301 (1999).

36. J. Flammer et al., "The probable involvement of factors other than ocular pressure in the pathogenesis of glaucoma," in Pharmacology of Glaucoma S. M. Drance et al., Eds., pp. 273-283, Williams \& Wilkins, Baltimore (1992).

37. U. Guthauser, J. Flammer, and F. Mahler, "The relationship between digital and ocular vasospasm," Graefes Arch. Clin. Exp. Ophthalmol. 226(3), 224-226 (1988).

38. A. J. Salerian, N. G. Saleri, and J. A. Salerian, "Brain temperature may influence mood: a hypothesis," Med. Hypotheses 70(3), 497-500 (2008).

39. M. Abreu de Souza et al., "3D thermal medical image visualization tool: integration between MRI and thermographic images," Annual Int. Conf. of the IEEE Engineering in Medicine and Biology Society. IEEE Engineering in Medicine and Biology Society. Annual Conf. 55835586 (2014).

40. A. Wirz-Justice, "Diurnal variation of depressive symptoms," Dialogues Clin. Neurosci. 10(3), 337-343 (2008).

41. M. Matsuda-Nakamura, S. Yasuhara, and K. Nagashima, "Effect of menstrual cycle on thermal perception and autonomic thermoregulatory responses during mild cold exposure," J. Physiol. Sci. 65(4), 339-347 (2015).

Jerome J. Maller is a neuroscientist and senior research fellow at the Monash Alfred Psychiatry Research Centre, Alfred and Monash University Central Clinical School, Melbourne, Victoria, Australia. With a background in brain imaging, neurostimulation, head injury, and psychiatry, he applies his abilities to develop new diagnostic tools and treatment strategies for psychiatric disorders and traumatic brain injury.

Shefin Sam George is a biomedical engineer with expertise in neuroscientific applications. Her current research focuses on enhancing psychiatric diagnosis through biomedical innovations and investigating focusing stimulation techniques for cochlear implants using electrophysiological studies.

Rekha Puzhavakkathumadom Viswanathan is an electronics engineer with a background in diagnosis and assessment of neurodegenerative disorders such as Parkinson's disease. Her current research focuses on the use of thermographic technology to investigate its potential to explore psychiatric conditions.

Paul B. Fitzgerald is a professor and deputy director of the Monash Alfred Psychiatry Research Centre. He has over 20 years of experience in clinical psychiatry and innovation in the fields of neuroimaging and brain stimulation technologies.

Paul Junor is a senior lecturer at La Trobe University in Victoria, Australia, and an electronics engineer with substantial experience in the field of biomedical science, particularly sensor technology. 